Meta

Journal des traducteurs

Translators' Journal

\title{
Sur les relations entre terminologie et lexique
}

\section{Michel Le Guern}

Volume 34, numéro 3, septembre 1989

1. Actes du Colloque Les terminologies spécialisées : Approches quantitative et logico-sémantique et 2 . Actes du Colloque Terminologie et Industries de la langue

URI : https://id.erudit.org/iderudit/003458ar

DOI : https://doi.org/10.7202/003458ar

Aller au sommaire du numéro

Éditeur(s)

Les Presses de l'Université de Montréal

ISSN

0026-0452 (imprimé)

1492-1421 (numérique)

Découvrir la revue

Citer cet article

Le Guern, M. (1989). Sur les relations entre terminologie et lexique. Meta, 34(3),

340-343. https://doi.org/10.7202/003458ar d'utilisation que vous pouvez consulter en ligne.

https://apropos.erudit.org/fr/usagers/politique-dutilisation/ 


\section{SUR LES RELATIONS ENTRE TERMINOLOGIE ET LEXIQUE}

MiChel Le GUERN

Université Lumière-Lyon 2, Lyon, France

L'intérêt et l'abondance des travaux actuels concernant la terminologie rendent possible et nécessaire l'élaboration des fondements théoriques sans lesquels cette discipline encore jeune aurait du mal à atteindre un statut pleinement scientifique. La question centrale, comme l'a montré Louis Gilbert dans son article «La spécificité du terme scientifique et technique» Langue française, no. 17, 1973, est celle des relations entre terminologie et lexique. Je voudrais signaler dès maintenant que, dans un cadre épistémologique tout à fait différent de celui de Louis Gilbert, j'aboutis aux mêmes résultats que lui. Ses vues trouveront ici une confirmation, et si l'on voulait interpréter ma contribution comme une reformulation quelque peu logicienne de ce qu'il a apporté, je n'y verrais pour ma part aucun inconvénient.

Il faut bien commencer par reconnaître que, pour beaucoup, le lexique et la terminologie ne sont séparés que par une frontière floue, quand ce n'est pas la confusion la plus totale. Confusion dont il est d'autant plus difficile de se défendre qu'elle s'appuie sur une plus longue tradition. Furetière, en qui on reconnaît parfois un précurseur des études terminologiques, porte une part de responsabilité dans cette confusion. Son Dictionnaire universel, publié en 1690, mêle le lexique et la terminologie comme le titre l'indique: «Dictionnaire universel, contenant tous les mots français tant vieux que modernes», voilà pour le lexique - «et les termes de toutes les sciences et des arts, savoir la philosophie, logique, et physique, la médecine, ou anatomie, pathologie, thérapeutique, chirurgie, pharmacopée, chimie, botanique», etc. (il y en a vingt lignes) - voilà pour la terminologie. Furetière, il est vrai, avait bien des excuses; il ne disposait pas encore des deux domaines. Aujourd'hui, notre situation n'est plus la même, et nous n'avons plus d'excuse pour prétendre que la terminologie est une partie du lexique, ou pour la définir comme «un lexique spécialisé».

Pour y voir clair, il suffit de ne pas confondre les mots et les choses. On peut dire que le lexique concerne les mots indépendamment des choses, alors que dans la terminologie, les mots sont liés aux choses. Mais d'un côté et de l'autre, ce ne sont pas les mêmes «mots». Ils ont bien l'air d'être les mêmes, et beaucoup de gens s'y trompent, mais l'objet «mot» pertinent pour le lexique est une réalité totalement distincte de l'objet «mot» qui appartient à la terminologie.

Le lexique d'une langue est constitué de l'ensemble des mots de cette langue. Mais les mots de la langue ne sont pas les mots du discours. La différence est évidente si l'on adopte la perspective de la sémiotique peircienne; dans la typologie de Peirce, les mots de la langue sont des légisignes symboliques rhématiques, alors que les mots du discours sont des sinsignes indiciaires rhématiques; il est impossible que les mots du discours soient symboliques, puisque ce sont des sinsignes, des occurrences singulières: les symboles sont nécessairement des légisignes.

Les mots de la langue ne sont pas en relation immédiate avec les choses. Ils ont un signifié, mais n'ont pas de référence. Le mot maison ne dit pas maison. Le mot cheval ne dit pas un cheval; il n'est en relation immédiate avec aucun être concret; il exprime seule- 
ment un ensemble de propriétés, et la question de savoir s'il existe dans l'univers des êtres auxquels appartiennent ces propriétés n'a aucune pertinence pour le lexique : c'est de la même manière que cheval et licorne font partie du lexique du français. Comme le fait Gustave Guillaume dans ses travaux sur l'article, il faut dénoncer l'illusion du substantif: le lexique de la langue ne contient pas de substantifs; les mots que les lexicographes désignent comme substantifs ne sont en réalité que des prédicats, ils disent des propriétés et non des substances, des qualités et non des objets.

Le signe d'une propriété est un prédicat, le signe d'un objet est un terme. Les termes ne font pas partie du lexique, c'est le discours qui les construit. Les mots que le lexicographe désigne comme substantifs ne sont pas en réalité des termes, des substantifs, en tant qu'ils font partie du lexique de la langue. C'est leur emploi dans le discours qui les distingue, par la position centrale qui leur est donnée dans la construction des termes. Pour désigner des objets, le discours ne met pas seulement en œuvre le lexique, il a aussi besoin de la syntaxe. L'unité minimale de discours qui a la possibilité de signifier un objet est le syntagme nominal. Maison, le mot du lexique, ne signifie aucune maison que ce soit, alors qu'il suffit que le discours construise le syntagme «une maison» pour que soit désigné un objet concret. La fermeture du prédicat par le quantificateur le transforme en terme. Mais on peut encore se demander si le prédicat que ferme le quantificateur est le même que celui qui appartient au lexique.

La logique du lexique n'est pas la logique du discours. Dans ce partage des logiques, je place la terminologie du côté du discours. La logique classique associe extension et compréhension, qui sont en relation inverse; cela est trop connu pour qu'on y insiste; il me suffit de renvoyer à La logique ou l'art de penser d'Antoine Arnauld et Pierre Nicole, Première Partie, chapitre 6. Cette logique s'applique incontestablement au discours, mais elle ne fournit pas un modèle adéquat à la représentation du lexique. Pour qu'on puisse parler d'extension, il faut considérer un référentiel, un univers constitué d'objets appartenant ou non à l'extension considérée. Or le lexique signifie indépendammant de quelque univers que ce soit: les éléments qui le constituent n'ont pas d'extension, ils n'ont qu'une compréhension, ou si l'on préfère le dire ainsi, une intension. À la logique extensionnelle du discours s'oppose la logique intensionnelle du lexique.

Mais le discours se construit avec les mots du lexique. Il faut donc rendre compte de la manière dont s'articulent dans cette construction les deux logiques opposées. Maison, comme mot du lexique, est un prédicat qui relève de la logique intensionnelle. «La maison de Paul» est un syntagme nominal, c'est déjà du discours; c'est un terme qui prend sa valeur sur un univers donné; il relève donc de la logique extensionnelle. Ce terme, «la maison de Paul», est analysable : il est obtenu par une opération de fermeture du quantificateur «la» sur le prédicat complexe «maison de Paul». Ce prédicat complexe ne fonctionne pas comme le prédicat maison, mot du lexique, et cette différence de fonctionnement n'est pas due au seul fait que l'un des prédicats est complexe et que l'autre ne l'est pas. Le prédicat «maison de Paul» a ceci de particulier qu'il suppose un univers donné, univers dans lequel existe une personne du nom de Paul. Appelons-le prédicat lié. En revanche, maison, mot du lexique, ne suppose aucun univers déterminé, pas plus d'ailleurs que pomme de terre ou montre à quartz, qui sont pourtant des prédicats complexes; ce qu'ils ont en commun, c'est de signifier en dehors de la prise en compte de quelque univers que ce soit. Appelons-les prédicats libres.

On peut maintenant décrire la manière dont se construit le syntagme nominal «une maison». Je prends dans le lexique le prédicat libre maison et je le place dans mon univers de discours; de ce fait, il n'est plus libre et devient un prédicat lié, et je suis passé dans une logique extensionnelle. Transformer un prédicat libre en prédicat lié, c'est lui associer une classe d'objets pris dans un univers déterminé. La quantification agit sur des 
classes, et non sur des prédicats libres. Il faut donc que les prédicats libres soient transformés en prédicats liés pour pouvoir être soumis à la quantification. On peut préciser maintenant les relations entre lexique et terminologie. Je reprends l'exemple de «maison» dans «une maison». Tant que je considère maison comme prédicat libre, je reste dans le lexique; dès que je l'envisage comme prédicat lié, je suis dans la terminologie.

Le mot lexique a un signifié, il n'a pas de référence: il exprime un ensemble de propriétés, indépendamment de quelque objet que ce soit situé dans quelque univers que ce soit. Le mot cheval, en tant qu'il appartient au lexique français, ne renvoie à aucun cheval, reel ou imaginaire, pas même au concept de cheval; il ne dit que la "caballéité», ensemble de propriétés abstraites qui ne coïncide pas avec la «horséité», ensemble de propriétés que signifie le mot horse dans le lexique de l'anglais. Un mot d'une langue ne peut pas se traduire en un mot d'une autre langue. Les lexiques sont intraduisibles. En revanche, la terminologie est constituée de prédicats liés, c'est-à-dire d'étiquettes de classes d'objets pris dans un univers donné. «Cheval», en tant qu'élément de la terminologie de l'univers des animaux domestiques, désigne une classe particulière d'animaux, la classe à laquelle correspond en anglais l'étiquette «horse». Le lexique considère les mots, la terminologie considère les choses. Il n'existe pas d'équivalence d'un mot du lexique d'une langue à un mot du lexique d'une autre langue. Mais, si l'on se place dans la perspective de la terminologie, la même classe d'objets d'un univers donné peut avoir une étiquette dans une langue et une étiquette dans une autre langue; dès lors, la traduction devient possible, fondée sur une synonymie référentielle. Si deux termes ont la même extension dans un univers donné, on peut les considérer comme équivalents et les traduire l'un par l'autre.

L'impossibilité de traduire les prédicats libres, ceux qui appartiennent au lexique, alors que les prédicats liés acceptent la traduction, peut apparaître comme un paradoxe. On peut se demander comment il se fait que deux mots, appartenant à des langues différentes, n'ont pas le même signifié, même si dans leur usage habituel ils servent à désigner la même classe d'objets. Il est facile d'en rendre compte dans le cadre de la sémantique componentielle. Dans une perspective cognitive, les objets et les classes d'objets s'opposent par des traits de substance, en nombre variable suivant les individus, puisque cela dépend du savoir, de l'expérience et de la culture de chaque personne. Les mots de la langue s'analysent en sèmes ou prédicats élémentaires. Le signifié d'un mot donné n'est pas constitué de la totalité des traits de substance associés à l'objet que ce mot désigne habituellement, mais il résulte d'un choix collectif, lié à l'histoire de la langue et à sa structure. Chaque langue fait son choix. Dans les langues du Togo, le mot qui désigne le lièvre signifie «rusé»; or, en français, le sème /rusé/ ne fait pas partie du signifié de lièvre; on aurait tort d'en inférer que les lièvres français sont moins malins que les lièvres africains : mes amis chasseurs $m$ 'ont rassuré sur ce point. Dans une langue du Gabon, le punu, le mot qui désigne la gazelle comporte le sème /intelligent/alors que celui qui désigne l'antilope comporte celui de /stupide/; cette opposition ne se retrouve pas dans les signifiés de gazelle et d'antilope en français. Alors que seuls les sèmes sont pertinents pour le lexique, les emplois en prédicats liés peuvent se charger, par un effet de contexte, des traits de substance des classes d'objets qu'ils désignent, même s'ils n'ont pas été retenus par la langue pour constituer les signifiants lexicaux. L'opposition entre sèmes et traits de substance permet aussi d'y voir un peu plus clair dans les théories componentielles: les sèmes de Greimas sont ceux que j'appelle sèmes, alors que ceux de Pottier sont plutôt du côté de mes traits de substance. Ce sont les traits de substance qui sont pertinents en terminologie et non les sèmes; quant au choix de ces traits de substance, chaque terminologie spécialisée l'établit en fonction du point de vue propre de sa disci- 
pline: le signifié lexical de loup contient le sème /méchant/, que la terminologie de l'éthologie animale ne retiendra sans doute pas comme trait de substance pertinent.

Un des aspects théoriques dont les conséquences pratiques découlent de la manière la plus évidente est celui de la délimitation des unités terminologiques, qu'il importe de distinguer des unités lexicales. Prenons comme exemple la paire d'étiquettes terminologiques «trompe d'Eustache», et «trompe de chasse», qui peuvent également prendre place dans le domaine spécialisé de l'audition. Le fait que l'élément «trompe» soit commun n'a aucune pertinence sur le plan de la terminologie; il ne correspond à aucune relation entre les classes d'objets désignées. «Trompe» n'est pas ici une unité terminologique. Ce qui ne l'empêche pas d'être une unité lexicale, dont le signifié exprime un prédicat commun, quelque chose comme «en forme de tube évasé». Un prédicat commun ne suffit pas à constituer une classe pertinente dans un domaine donné, ce qui se traduit par le fait qu'une unité lexicale peut ne pas constituer une unité terminologique. Inversement, «reproduction des kangourous dans les parcs zoologiques» constitue une unité terminologique, non une unité lexicale; c'est encore vrai pour «reproduction des kangourous». Cela provient de ce que «reproduction des kangourous» ne peut être qu'un prédicat lié, non un prédicat libre, du fait qu'il domine un syntagme nominal. N'y font exception que les lexies figées, du type de «rose des vents» qui n'a plus grand chose à voir ni avec les roses ni avec les vents; «les vents» n'y constitue plus qu'un pseudo-syntagme nominal, puisqu'il n'a plus de référence.

Le lexique et la terminologie sont constitués pour l'essentiel de prédicats complexes. Le fait que l'on ait d'un côté des prédicats libres et de l'autre des prédicats liés n'empêche pas qu'il soit possible de rendre compte de la composition des prédicats complexes de l'un et de l'autre type par le même modèle, celui de la logique combinatoire de Curry. On peut considérer un prédicat complexe comme le résultat de l'action d'un prédicat opérateur sur un prédicat opérande. Si le prédicat opérateur et le prédicat opérande sont tous deux des prédicats libres, le résulat est un prédicat libre; en revanche, il suffit qu'un des deux prédicats, l'opérateur ou l'opérande, soit lié, pour que le résultat soit nécessairement un prédicat lié. Le choix d'un modèle commun pour le lexique et la terminologie a le double avantage de bien rendre compte à la fois de ce qui les rapproche et de ce qui les distingue.

Parmi les problèmes que permet de résoudre la confrontation entre lexique et terminologie, il y a aussi celui de la relation entre signifié et concept: le signifié est du côté du lexique, le concept du côté de la terminologie; pour la démonstration, je la remets à une autre fois. 